\title{
Seleção Dinâmica da Dimensão do Subespaço de Krylov no Método GMRES $(m)$ e suas Variantes
}

T.T. GONÇALEZ, Programa de Pós-Graduação em Matemática Aplicada, Universidade Federal do Rio Grande do Sul, Porto Alegre, RS, Brasil.

R.D. DA CUNHA ${ }^{1}$, Instituto de Matemática, Universidade Federal do Rio Grande do Sul, Porto Alegre, RS, Brasil.

Resumo. Nesse trabalho apresentamos alguns algoritmos adaptativos do Método do Resíduo Mínimo Generalizado (GMRES) [10], um método iterativo para resolver sistemas de equações lineares com matrizes não simétricas e esparsas, o qual baseiase nos métodos de projeção ortogonal sobre um subespaço de Krylov.

O GMRES apresenta uma versão reinicializada, denotada por $\operatorname{GMRES}(m)$, também proposta em [10], com o intuito de permitir a utilização do método para resolver sistemas de equações lineares cuja matriz dos coeficientes apresenta uma grande dimensão. No entanto, escolher um valor apropriado, $m$, para a dimensão da base do subsespaço de Krylov é bastante difícil.

Dessa forma, nesse trabalho, acrescentamos ao $\operatorname{GMRES}(m)$ e algumas de suas variantes um critério que tem por objetivo escolher, ao longo das iterações, um $m$ tal que se obtenha a convergência do método, possivelmente de forma mais rápida.

Aproximadamente duas centenas de testes foram realizados utilizando as matrizes da coleção Harwell-Boeing, que foram utilizados para mostrar o comportamento dos algoritmos adaptativos. Foram obtidos resultados muito bons conforme apresentaremos nesse trabalho.

\section{Introdução}

Nesse trabalho são estudados aprimoramentos ao método iterativo "Generalized Minimum Residual" (GMRES), proposto por Saad e Schultz em 1986 [10], e algumas de suas variantes, utilizados na solução de sistemas de equações lineares da forma

$$
A x=b,
$$

onde $A \in \mathbb{R}^{n \times n}$ é não-simétrica e esparsa e $x, b \in \mathbb{R}^{n}$. No GMRES original, conforme proposto por Saad e Schultz, o resíduo inicial $r_{0}=b-A x_{0}$ é utilizado para gerar uma base ortonormal para um subespaço de Krylov,

$$
\mathcal{K}_{k}\left(A, r_{0}\right)=\operatorname{span}\left(r_{0}, A r_{0}, \ldots, A^{k-1} r_{0}\right),
$$

\footnotetext{
${ }^{1}$ rudnei.cunha@ufrgs.br
} 
onde $k$ é o número de iterações do método GMRES, através do processo de Arnoldi, o qual é uma modificação do processo de ortonormalização modificado de GramSchimidt. A solução do sistema acima é tomada como solução de um problema de mínimos quadrados que envolvem uma matriz e um vetor gerados durante o processo de Arnoldi. Dentre algumas características do GMRES, destaca-se a convergência em, no máximo, $n$ iterações. Obviamente, para $n$ muito grande isso não é uma vantagem, pois o processo de Arnoldi requererá o armazenamento de $n$ vetores de dimensão $n$, além de uma matriz de dimensão $(n+1) \times n$ - o que levará a uma complexidade de ordem $O\left(n^{2}\right)$ - e exigirá $n$ produtos matriz-vetor. Dessa forma em 1986, Saad e Schultz propuseram uma versão chamada reinicializada, $\operatorname{GMRES}(m)$, onde $m$ é a dimensão da base do subespaço de Krylov, na qual se produz uma base ortonormal para o subespaço de Krylov de dimensão $m, \mathcal{K}_{m}\left(A, r_{k}\right)$; isto é, a cada iteração $k$, calculam-se o resíduo $r_{k}=b-A x_{k}$ e os $m$ vetores ortonormais através do processo de Arnoldi e, a partir deles resolve-se um problema de minimos quadrados, obtendo-se uma aproximação $x_{k+1}$ para a solução do problema. Infelizmente, ao se optar pelo método GMRES $(m)$, perde-se a garantia de convergência apresentada pelo método GMRES, a menos que $m$ seja suficientemente grande. Em [10] foi proposto o seguinte teorema para a escolha de $m$ :

Teorema 1.1. Suponha que A é diagonalizável. Assuma que existem $\nu$ autovalores $\lambda_{1}, \lambda_{2}, \ldots, \lambda_{\nu}$ de $A$ com parte real não positiva e tome os outros autovalores sendo fechados em um círculo centrado em $C$, com $C>0$ e tendo raio $R$ com $C>R$. Então, o GMRES(m) converge para algum valor inicial de $x_{0}$ se

$$
m>\nu \frac{\log \left[\frac{D C}{d R} \kappa(X)^{\frac{1}{\nu}}\right]}{\log \frac{C}{R}},
$$

onde

$$
D=\max _{i=1, \nu ; j=\nu+1, n}\left|\lambda_{i}-\lambda_{j}\right|, \quad d=\min _{i=1, \nu}\left|\lambda_{i}\right| .
$$

Demonstração. Ver [10].

Podemos perceber que a determinação desse valor mínimo $m$ depende da determinação da distribuição dos autovalores de $A$ e assim é usado somente em casos especiais, não podendo ser tomada como padrão na escolha do $m$.

Para comprovar que nem todas as matrizes satisfazem tal teorema, geramos uma matriz utilizando o programa MATLAB 5.3, a qual satisfaz as condições do teorema apresentado acima. Apresentamos os gráficos da estrutura e dos autovalores de tal matriz, na Figura 1. Para $n=100$ obtivemos os seguintes valores para os parâmetros do teorema: $D=192,5682 ; d=54,0479 ; C=3,0004 ; R=1,4140 ; \nu=17$, tal que $m>84,8287$. Utilizando a mesma matriz no método GMRES $(m)$ obtivemos $m=20$. Observe que o valor de $m$ proposto pelo teorema é muito superior ao necessário para a resolução do sistema através do $\operatorname{GMRES}(m)$. Isso mostra que tal teorema não é adequado e, particularmente para as matrizes utilizadas nos testes que serão apresentados nesse trabalho, a distribuição dos autovalores das mesmas não satisfaz a hipótese do teorema. Em [5] são apresentados gráficos da 

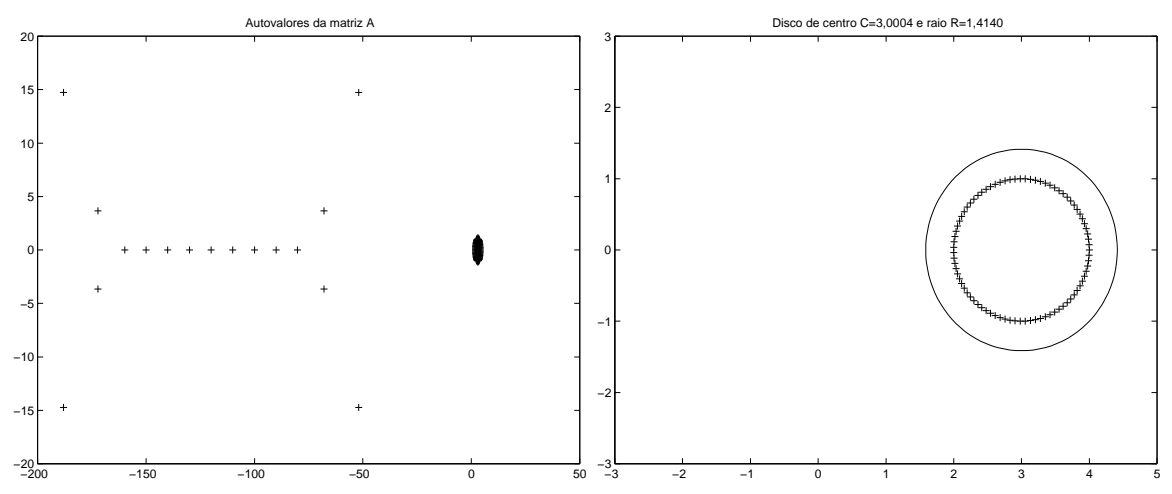

Figura 1: a) Autovalores da matriz, b) Detalhe ampliado.

distribuição de autovalores de várias matrizes armazenadas em [8], e para nenhuma delas o teorema é satisfeito. Em vista disso, tal teorema torna-se muito limitado, tornando-se necessário uma estratégia mais abrangente e que não apresente tantas limitações quanto às características da matriz.

Outros resultados referentes à convergência do GMRES foram propostas por Joubert [7] e Embree [4]. Joubert propõe um algoritmo adaptativo para o $\operatorname{GMRES}(m)$, no sentido de efetuar a reinicialização apenas quando a convergência não é considerada adequada, de acordo com um critério envolvendo a taxa de convergência ([7, p. 440]), porém sem alterar o valor de $m$ ao longo das iterações. Já os resultados propostos por Embree utilizam quantidades como o número de condição e "field of values", cujo cálculo aumentaria consideravelmente o custo computacional de se resolver um sistema linear; porém, esses resultados não apresentam um procedimento para a seleção de um valor mais adequado para $m$. Dessa forma, concluímos que nem o teorema proposto em [10] nem as condições propostas em [4] são adequados para a escolha e/ou correção do valor de $m$.

Nesse trabalho apresentamos estratégias para determinar dinamicamente, ao longo do processo de solução do sistema $A x=b$, um valor adequado para $m$, de forma a garantir a convergência do método $\operatorname{GMRES}(m)$ e de algumas de suas variantes, bem como obter a solução com o menor gasto computacional possível, em termos de número de operações de ponto-flutuante.

\section{Descrição dos Métodos}

Os experimentos foram realizados utilizando-se o método $\operatorname{GMRES}(m)$; uma variante do $\operatorname{GMRES}(m)$ utilizando transformações de Householder, ao invés do processo de Arnoldi, proposta por Walker [12], a qual chamaremos de GMRESH $(m)$; o "Simpler GMRES"e sua variante usando transformações de Householder [13], os quais chamaremos de $\operatorname{SGMRES}(m)$ e SGMRESH $(m)$; o "LooseGMRES", proposto Baker et al. [1], chamados de $\operatorname{LGMRES}(m)$ e $\operatorname{LGMRESH}(m)$; o GMRES adaptativo 
proposto em [3], chamado de A-GMRES $(m)$; e o $\operatorname{BC}-\operatorname{GMRES}(m)$, proposto em [9]. Apresentamos agora uma breve explicação das variantes do GMRES, com suas principais características e vantagens.

O GMRESH $(m)$ apresenta melhores propriedades numéricas que o $\operatorname{GMRES}(m)$, especialmente nas iterações finais, quando os resíduos tornam-se cada vez mais próximos, e utiliza menos armazenamento, apesar de requerer duas vezes mais operações aritméticas do que o $\operatorname{GMRES}(m)$. Segundo [12], em $\operatorname{GMRESH}(m)$ os vetores de Householder têm dimensão menor e, junto com a matriz triangular superior gerada pelo algoritmo, podem ser armazenados em uma matriz de ordem $n \times($ maxit +1$)$, onde maxit é o número máximo de iterações. Isto é um pouco menos armazenamento do que o requerido pelo $\operatorname{GMRES}(m)$, o qual armazena toda a base de vetores ortonormal, bem como as matrizes triangulares superiores. Assim, como em geral temos que maxit $\ll n$, então essa seria a maior vantagem entre esses dois algoritmos.

O Simpler GMRES surgiu em 1994 com Homer F. Walker e Lu Zhou [13], sendo apresentado com uma modificação no processo de Arnoldi original do GMRES. Segundo [13], na implementação usual do GMRES, o processo de Arnoldi é aplicado $\operatorname{com} v_{1}=\frac{r_{0}}{\left\|r_{0}\right\|_{2}}$. A ortogonalização em cada passo do algoritmo pode ser feita usando o processo modificado de Gram-Schmidt ou transformações de Householder. Note que o SGMRES é resultante apenas da modificação feita no $v_{1}$, tornando assim o problema de mínimos quadrados em um problema de mínimos quadrados triangular superior, eliminando a fase de triangularização da matriz de Hessenberg.

O Loose GMRES surgiu em 2003 com Baker, Jessup e Manteuffel; veja [1]; sendo apresentado com uma técnica para acelerar a convergência do GMRES. O LGMRES $(m, k)$ é muito semelhante ao método do gradiente conjugado completo (CG) com precondicionador polinomial, e sua implementação não exige muitas modificações no $\operatorname{GMRES}(m)$. Segundo [1], uma motivação para propor o LGMRES se deu pelo fato de que o $\operatorname{GMRES}(m)$ não mantém a ortogonalidade entre espaços aproximados generalizados em sucessivas reinicializações, tornando assim sua convergência vagarosa ou até mesmo levando a divergência. Também é sabido, conforme [2], que métodos aumentados formam uma classe de técnicas de aceleração; esses métodos buscam evitar a divergência melhorando assim as informações obtidas com o GMRES em cada reinicialização. Tipicamente, um subespaço A-invariante é agrupado ao subespaço aproximado de Krylov, resultando assim em um subespaço de Krylov aumentado. O subespaço A-invariante associado com os menores autovalores é comumente usado. A idéia então consiste de adicionar vetores ao subespaço aproximado de Krylov que representaria o espaço aproximado para cada ciclo de reinicialização. O LGMRES $(m, k)$ tipicamente não requer mais iterações do que o $\operatorname{GMRES}(m)$, e o fato do mesmo ser um acelerador de uma possível convergência do $\operatorname{GMRES}(m)$ não significa que o mesmo não possa ser aplicado sem o uso de pré-condicionadores.

O método GMRES-Adaptativo foi proposto por Maria Sosonkina Driver em 1997 [3], e o denotamos por A-GMRES. A diferença básica em relação ao $\operatorname{GMRESH}(m)$ consiste no fato de que o A-GMRES apresenta, se necessário, alguns testes para incrementar o $m$ e então reinicializar o método. De acordo com [3] a essência do A-GMRES é obter um parâmetro $m$ adequado para a reinicialização do problema. 
O BC-GMRES foi proposto por Takashi Nodera e Kentaro Moriya em 2003 [9], e é um método que escolhe o ciclo de reinicialização do $m$ baseado no teste da norma residual e na distribuição dos zeros do polinômio residual do GMRES $(m)$. Essa estratégia foi baseada em algumas conclusões apresentadas em [11]. O BCGMRES executa a reinicialização quando a distribuição dos zeros do polinômio residual do $\operatorname{GMRES}(m)$ tornam-se ideais, ou seja, pode-se dizer que quanto mais espalhados estão os zeros do polinômio residual mais provável que a distribuição dos zeros aproximados seja similar aos zeros ideais. No BC-GMRES a decisão da reinicialização é tomada através da análise de duas condições, a saber: a distribuição dos zeros aproximados e o teste de convergência da norma residual.

\section{Critério de Escolha do Valor de $m$}

Apresentamos agora o critério desenvolvido para a escolha de $m$ adequado, sendo que os objetivos para a elaboração do tal critério foram a redução da norma residual e a variação de $m$, ou seja, buscamos um procedimento que possa determinar o aumento ou a redução de $m$. Inicialmente tentamos desenvolver um critério que apresentasse resultados satisfatórios quanto ao número de convergência dos métodos adaptativos e ao número de não convergências.

Foram elaborados cinco critérios ao longo de todo o trabalho, sendo que os três primeiros utilizavam o número de dígitos significativos exatos (DIGSE) como ferramenta para a comparação da variação do $m$. A idéia aqui era determinar a quase estagnação da convergência através das normas de dois resíduos sucessivos. Conforme pode-se observar em [5], os critérios elaborados com o DIGSE não apresentaram resultados satisfatórios, visto que o $m$ praticamente não apresentou variação. Optou-se então por alterar o valor de $m$ de acordo com o logaritmo (base 10) das normas dos resíduos, e estabelecendo faixas de variação dentro das quais alteraríamos o valor de $m$ de forma adequada. Por exemplo, se $\log \|r\|_{2}>0$, então o valor de $m$ é duplicado (até um certo limite limite pré-especificado $m_{\max }$, o qual pode ser escolhido em função de fatores como a memória disponível, por exemplo). Se $\log \left\|r_{i}\right\|_{2}<0$ e $\log \left\|r_{i}\right\|_{2}>\frac{2 \log \epsilon}{3}$, então o processo já está convergindo, e cabe agora verificar se está convergindo lentamente, caso em que adiciona-se a $m$ o valor de $m_{\text {orig }}$ (o valor inicialmente estipulado para $m$ ); caso contrário, podemos reduzir o valor de $m$, subtraindo dele $\frac{m_{\text {orig }}}{3}$ (desde que $m$ nunca seja menor do que $m_{\text {orig }}$ ). Se $\log \left\|r_{i}\right\|_{2}<0$ e $\log \left\|r_{i}\right\|_{2}<\frac{2 \log \epsilon}{3}$, então o método já está quase que alcançando a tolerância especificada, e pode-se ainda alterar o valor de $m$, porém com quantidades menores, pois pode ser dispendioso aumentar por demais $m$, já que a convergência quase foi alcançada. Por isso, quando a norma do resíduo encontra-se nessa faixa, porém a taxa de convergência é lenta, então adiciona-se a $m$ o valor de $\frac{m_{\text {orig }}}{2}$; caso contrário, reduzimos o valor de $m$, subtraindo dele $\frac{m_{\text {orig }}}{4}$.

Em nosso critério, utilizamos como fator de determinação da taxa de convergência do método a relação $\frac{\left\|r_{i-5}\right\|_{2}}{\left\|r_{i}\right\|_{2}}$ e dizemos que ela é "rápida" se tal relação for superior a 2. Além disso, a fim de evitar muitas mudanças no valor de $m$, optamos por alterá-lo, se necessário, a cada cinco iterações. Tal idéia é baseada no algoritmo adaptativo para escolha do parâmetro de relaxação do método SOR conforme pro- 
posto por Hageman e Young em 1981 [6]. O critério de escolha do valor de $m$ pode ser apresentado de forma algorítmica como segue:

Algoritmo 3.1. Critério de escolha de $m$ :

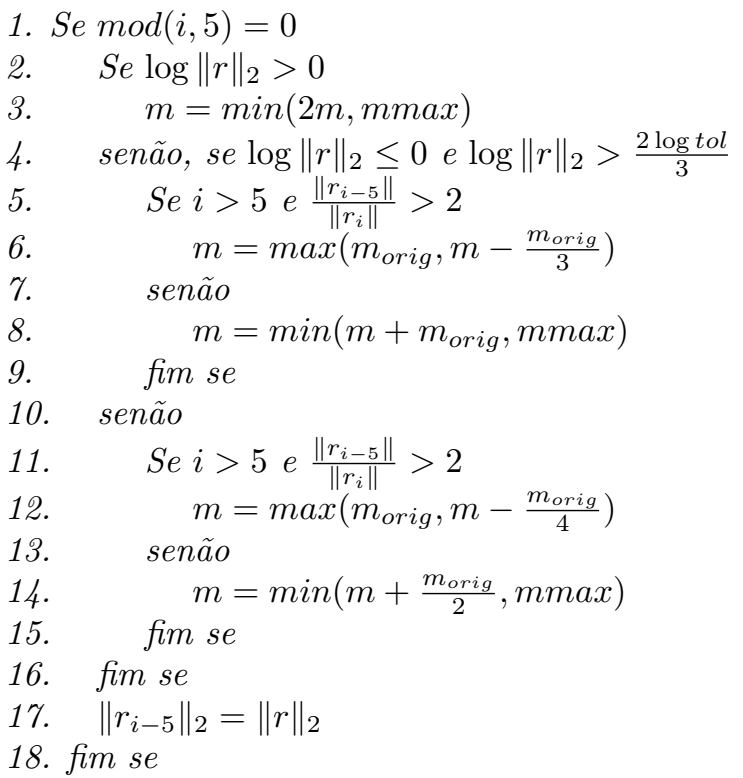

Esse critério foi embutido em todos as variantes do GMRES apresentadas na Seção 2, a cujas abreviações foi adicionada a letra A para indicar o uso do critério. Os métodos foram comparados como segue: SGMRES-A $(m)$ com $\operatorname{SGMRES}(m)$; $\operatorname{SGMRESH}-\mathrm{A}(m)$ com SGMRESH $(m)$; BC-GMRES $(m)$ com BC-GMRES-A $(m)$; $\operatorname{LGMRES-A}(m, k)$ com LGMRES $(m, k)$ e, por fim, o A-GMRES $(m)$ com o método $\operatorname{GMRESH}(m)$, já que estes dois utilizam a formulação do $\operatorname{GMRES}(m)$ com base nas transformações de Householder.

Os métodos utilizados para a realização dos testes foram empregados sem précondicionadores e utilizou-se como critério de parada $\left\|r_{i}\right\|_{2}<\epsilon$, sendo $\epsilon=10^{-10}$. Os valores $m_{\text {orig }}$ e $m_{\max }$ foram estipulados como 10 e 50, respectivamente (o que representa um intervalo de valores típicos para $m$ encontrados na literatura).

Os testes foram realizados utilizando o software MATLAB versões 5.3 e 6.2 em um computador PC com processador AMD Athlon XP 2.400 e 512 MBytes de memória RAM. Ao todo foram realizados 114 testes, com as seguintes matrizes: ADD32, ARC_130, CAVITY05, CDDE1, E05R0000, E05R0300, FIDAPM05, FS_183_1, FS_760_1, GRE115, IMPCOL_A, JPWH991, NOS3, ORSIRR1, PDE900, SAYLR3, SHERMAN1 e STEAM1, presentes na coleção "Matrix Market" [8]. Para fins de explanação, apresentamos os gráficos comparativos da evolução da convergência do método adaptativo com o seu equivalente não-adaptativo, para as matrizes CDDE1 e E05R0300; além disso, num outro gráfico apresentamos a variação do valor de $m$ juntamente com a curva de $\log \left\|r_{i}\right\|_{2}$. Salientamos que o eixo horizontal, o qual representa as iterações, apresenta escalas diferentes em cada 

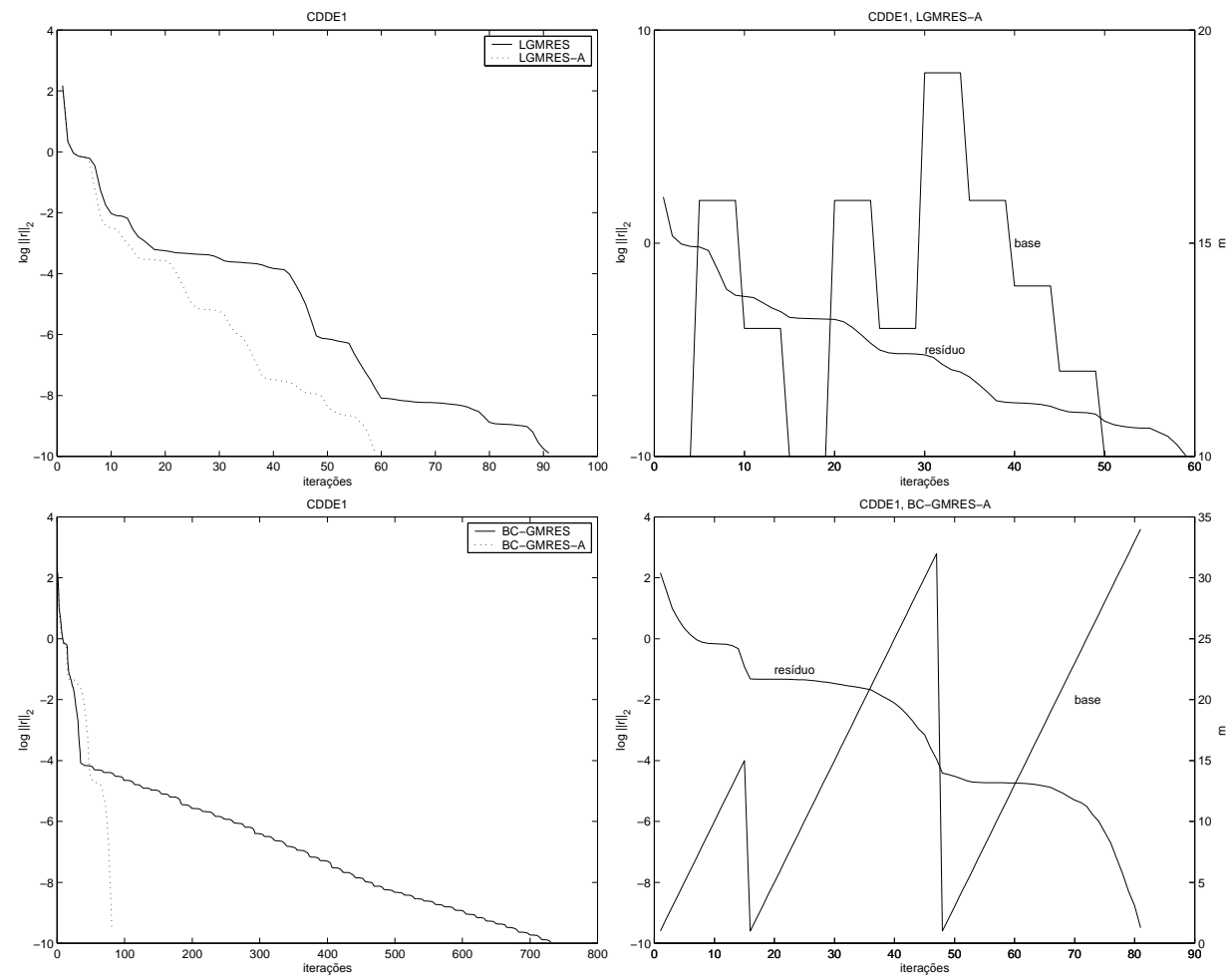

Figura 2: Curvas residuais para o problema CDDE1: acima, usando $\operatorname{LGMRES}(m, 2)$ e variação de $m$ e a curva residual para o método adaptativo LGMRES-A $(m, 2)$; abaixo, usando BC-GMRES $(m)$ e variação de $m$ e a curva residual para o método adaptativo BC-GMRES-A $(m)$.

método. As Figuras 2 e 3 ilustram o comportamento dos métodos usando o critério adaptativo.

Observe que o método adaptativo obteve a convergência em menos iterações e como, sempre que $m$ aumenta, há uma correspondente diminuição no valor do resíduo. Além disso, na Figura 2 observa-se uma rápida diminuição no valor da norma do resíduo, para a variante adaptativa do método BC-GMRES $(m)$ (cabe ressaltar que o perfil de dente-de-serra para a dimensão da base é típica do método BC-GMRES, já que ao realizar uma reinicialização no $\operatorname{GMRES}(m)$ a base inicia em 1). Na Figura 3, referente ao problema E05R0300, observa-se um comportamento atípico, dentre todos os testes realizados, pois não se obteve convergência com qualquer dos métodos, nem tampouco foi o critério adaptativo capaz de garantir convergência.

A Tabela 1 apresenta os resultados obtidos com todos os métodos estudados, para esse problema, onde pode-se observar que as versões adaptativas foram sempre melhores do que as não-adaptativas, garantindo a convergência e com menor custo 

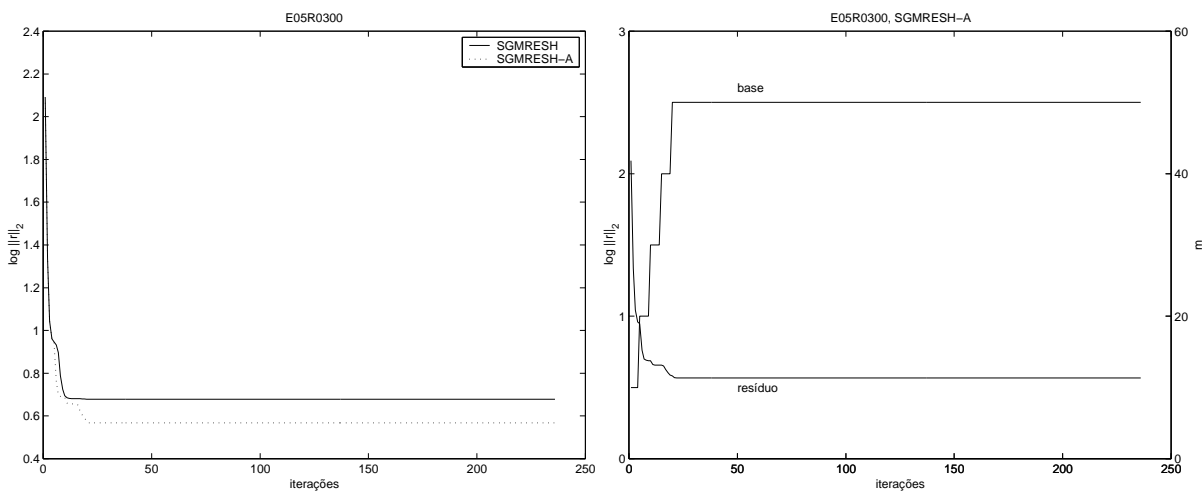

Figura 3: Curvas residuais para o problema E05R0300, usando $\operatorname{SGMRESH}(m)$ e variação de $m$ e a curva residual para o método adaptativo $\operatorname{SGMRESH-A}(m)$.

computacional.

Podemos resumir os resultados dos experimentos, tabelados em [5, pp.63-163], da seguinte forma: 76 testes convergiram para os métodos adaptativos, ou seja, para os métodos acrescidos do critério desenvolvido nesse trabalho para a escolha do $m$ adequado ao sistema estudado, apresentando menor número de flops e de iterações do que os demais; 24 métodos não adaptativos, ou seja, métodos que não apresentam nenhum critério para a escolha do $m$, convergiram apresentando menor número de flops do que as versões adaptativas e 12 testes não apresentaram convergência dentro da tolerância pré-especificada, em nenhum dos casos.

Dentre os valores apresentados devemos destacar que, para algumas matrizes, as versões adaptativos mantiveram-se absolutas, ou seja, todos os métodos adaptativos convergiram e apresentaram menor número de flops do que os demais, para as matrizes CDDE1, GRE115, NOS3, ORSIRR1, SAYLR3 e SHERMAN1. Em outros, pode-se notar que apenas as versões adaptativas obtiveram a convergência dentro da tolerância pré-especificada, como para FS_183_1; GRE115; LNS_131 e STEAM1, o que indica que o uso das versões adaptativas é indicado. Podemos citar ainda que entre 114 testes realizados, 5 apresentaram convergência apenas para as versões adaptativas, nenhuma apenas para as versões não-adaptativas, e dentre todos os testes, somente 2 sistemas não obtiveram a convergência em nenhum dos casos. Salientamos que, para os sistemas utilizando as matrizes E05R0300 e a IMPCOL_A, não se obteve convergência em nenhuma das versões; e, nos testes realizados com a matriz ARC_130, alguns métodos apresentaram o mesmo número de flops para as versões adaptativas e não adaptativas, fazendo com que não pudéssemos concluir nada a respeito das diferenças apresentadas por essas versões.

Um fato interessante foi que o número de iterações necessárias para obter a convergência das versões adaptativas sempre foram menores do que as demais; isso, independentemente, do número de flops apresentados pelos métodos. 


\begin{tabular}{ccccc}
\hline Método & $i$ & $m$ mínimo & $m$ máximo & Flops \\
\hline A-GMRES & 400 & 3 & 10 & $5,0780 \times 10^{8}$ \\
GMRESH-A & 30 & 2 & 20 & $9,5554 \times 10^{7}$ \\
SGMRES & 1000 & 7 & 10 & $3,5790 \times 10^{8}$ \\
SGMRES-A & 30 & 10 & 40 & $4,2707 \times 10^{7}$ \\
SGMRESH & 400 & 2 & 10 & $3,2589 \times 10^{8}$ \\
SGMRESH-A & 25 & 10 & 20 & $4,4876 \times 10^{7}$ \\
BC-GMRES & 1000 & 10 & 10 & $1,8685 \times 10^{8}$ \\
BC-GMRES-A & 70 & 10 & 60 & $3,8236 \times 10^{7}$ \\
LGMRES $(m, 2)$ & 35 & 10 & 10 & $1,6624 \times 10^{7}$ \\
LGMRES-A $(m, 2)$ & 14 & 10 & 20 & $1,4284 \times 10^{7}$ \\
LGMRES $(m, 3)$ & 45 & 10 & 10 & $3,9552 \times 10^{7}$ \\
LGMRES-A $(m, 3)$ & 40 & 10 & 20 & $2,3118 \times 10^{7}$ \\
\hline
\end{tabular}

Tabela 1: Resultados dos métodos para o problema CDDE1.

\section{Conclusão}

Em virtude dos dados apresentados podemos concluir que as versões adaptativas formam uma excelente alternativa para a resolução de sistemas não-simétricos esparsos, visto que tendem a diminuir o custo computacional e o tempo de execução dos mesmos.

Cabe ressaltar que os testes foram realizados sem o uso de pré-condicionadores, visto que o nosso objetivo nesse trabalho era de elaborar um critério adequado para a escolha da dimensão do subespaço de Krylov, em relação ao método GMRES em si. Não obstante, testes realizados posteriormente demonstraram que précondicionadores (como fatorações incompletas, polinomiais e outros) podem ser usados em combinação com o critério aqui proposto, com resultados semelhantes aos aqui relatados.

\section{Agradecimentos}

Os autores agradecem aos revisores pelas observações feitas, as quais contribuíram para a melhoria deste artigo.

\section{Referências}

[1] A. Baker, E. Jessup, T. Manteuffel, A Technique for Accelerating the Convergence of Restarted GMRES. Technical Report CU-CS-945-03, Dept. of Computer Science, College of Engineering and Applied Science, University of Colorado at Boulder, 2003.

[2] A. Chapman, Y. Saad, Deflated and augmented Krylov subspace techniques. Numerical Linear Algebra with Applications, 4 (1997), 15-41. 
[3] M.S. Driver, Parallel sparse linear algebra for homotopy methods. PhD thesis, Faculty of the Virginia Polytechnic Institute and State University, 1997.

[4] E. Embree, How descriptive are GMRES convergence bounds? Research Report NA-99/08, Numerical Analysis Group, Oxford University Computing Laboratory, 1999.

[5] T.T. Gonçalez, Algoritmos adaptativos para o método GMRES $(m)$. Dissertação de mestrado, Programa de Pós-Graduação em Matemática Aplicada, UFRGS, 2005.

[6] L.A. Hageman, D.M. Young, "Applied Iterative Methods", Academic Press, New York, 1981.

[7] W. Joubert, On the convergence behavior of the restarted GMRES algorithm for solving nonsymmetric linear systems. Numerical Linear Algebra with Applications, 1 No. 5 (1994), 427-447.

[8] Mathematical and Computational Sciences Division, Information Technology Laboratory, National Institute of Standards and Technology, Matrix Market: A visual repository of test data for use in comparative studies of algorithms for numerical linear algebra. http://math.nist.gov/MatrixMarket/, 2003.

[9] K. Moriya, T. Nodera, New adaptive $\operatorname{GMRES}(m)$ method with choosing suitable restart cycle $m$. In R. Wyrzykowski et al., editor, Parallel Processing and Applied Mathematics 2003, number 3019 in Lecture Notes in Computer Science, pp 1105-1113, Berlin, 2003. Spring-Verlag.

[10] Y. Saad, M.H. Schultz, GMRES: a generalized minimal residual algorithm for solving nonsymmetric linear systems. SIAM Journal of Scientific and Statistical Computing, 7 (1986), 856-869.

[11] N. Tsuno, T. Nodera, The speedup of the GMRES $(m)$ method using the early restarting procedure. Journal of the Information Processing Society of Japan, 40, No. 4 (1999), 1760-1773.

[12] H.F. Walker, Implementation of the GMRES method using Householder transformations. SIAM Journal on Scientific and Statistical Computing, 9, No. 1 (1989), 152-163.

[13] H.F. Walker, L. Zhou, A simpler GMRES. Linear Algebra and its Applications, 1 (1994), 574-581. 